\title{
Analysis of the Robustness of Enterprise Organization Based on Complex Network Perspective
}

\author{
Zhao Li \\ School of Management, Harbin University of Commerce, Harbin, China \\ Zhaoli0515@163.com
}

Keywords: complex network; enterprise organization; robustness

\begin{abstract}
Business organization as a complex system, its robustness and fragility which impact on the survival and development of enterprises directly. This paper looks for the evolution of complex network and constructs the model of business organization by abstracting enterprise organizations, then derivate and calculate their robustness and fragility, and obtain the robustness threshold of enterprise which under the random attacks and deliberate attacks, so as to draw a conclusion as follows: the enterprise is a scale free network when it reach a certain scale, its stability depends on the number of junior staff interaction.
\end{abstract}

\section{Introduction}

In recent years, robustness is a fundamental property of the system, which is a widespread phenomenon accompanied with uncertainty, as become the hot topics concerned by natural sciences, social sciences and other fields[1]. Overall, with respect to the domestic and foreign study of complex systems robustness has made a significant progress both in theoretical and empirical aspects, which results change and expand our understanding about variety of complex networks function and nature in large part. On the current literature, complex network theory has been applied to the study of social complex systems successfully, but research contents only limited on the analysis of structural characteristics of different types of complex networks and description of the robust performance, but the robustness measure of complex systems is rarely involved, especially study for the complex systems such as enterprise organization is relatively less. This paper tries to look for the evolution of complex network and constructs the model of enterprise organization from a new perspective, then derivation and calculate their robustness and fragility, and obtain the robustness threshold of enterprise organization which under random attacks and deliberate attacks.

\section{Evolution characristics of enterprise organization and Model construction}

Enterprise Organization development initially started by several people or a dozen people, at this stage there is no specific division of labor between them, but shared with all of the business work. Thus, the Phenomenon occurs that a person might act as several roles, and even one or two people compose a department. But as the enterprise capital accumulation and the business increasing, enterprise organization establish new departments ,even set up subsidiaries through recruiting new employees continuously, its scale is expanding continually[2]. Therefore, we can abstract the 
development process of enterprise as a complex network evolution, that is abstract all employees throughout the organization as the network node, and take the capital flow, information flow, logistics as the network edge. Thus, organizations can be seen as a evolving complex net works which composed by staff as nodes and resource flow as edges.

Evolution characteristics of Enterprise Organization. Suppose there are $m$ isolated departments initially during the development process of organizations and $m_{0}$ staff in each department, these employees interact through $e_{0}$ kinds of work flows. As the organization continues to expand, and its evolution process is as follows:

(1) Because of the nature of enterprise transformation, the organization will add a new department. A sector joining in organization affected by a variety of factors, including: leader decision, but sometimes the decisions are not entirely rational; and the flow topological properties of the workflow within the organization is changing continuously, that is resource flow changes continuously, and this flow of resources with a certain randomness. Since the department establishment is closely related to changes of external environment, so the process of the new department establishment is random, that is adding a department which has $m_{0}$ employees and $\boldsymbol{e}_{\mathrm{o}}$ edges by probability $\boldsymbol{P}$. Because the number of people in each department is very small during the early development of the business, with the time going, the number will gradually become larger, and consistent with other departments.

(2) As the business continues to increase, individuals and organizations whose function are also increasing, so every department of company may recruit new employees at any time. Employees will interact with others through resource flow after they entering into a particular department. This interaction between employees affected by the number of resource flow occupied by a particular staff in this sector (Such as department heads will make skilled employees have more information, knowledge and commit to more business.). Assumed that a new employee joins in a department, he and other staff in the same department will establish $m_{1}$ edges. First select a department randomly, the interaction between the new employees and other employees determined by the following probability:

$\Pi\left(k_{i}\right)=\frac{k_{i}}{\sum_{j \in \Omega} k_{j}}$

$\Omega$ represents a particular department. Repeat this process $m_{1}$ times.

(3) With the volume of business increasing, which resources flow growing increasingly. Therefore, it may add $\boldsymbol{m}_{2}$ resources flow to a particular sector. Because resources flow increasing are combine affected by organization external factors (such as market change) and internal factors (such as management level),so one side of the edge can be selected randomly. Since the node which occupies more resources flow is easier to get the new flow of resources, therefore, the other side of the edge can be selected with probability. Repeat this process $\boldsymbol{m}_{2}$ times.

(4) Similarly, due to it influenced by various external factors of the system, the business of a department will continue to reduce and its flow of resources will also be declining. So you can remove $m_{3}$ edges of a sector, and its edge selection can be accordance with the rules of plus side principles in

(3). ${ }_{3}$ Rtipd this process

(5) Since various departments will also interact through resource flow, so it may build $m_{4}$ edges between the selected one of the departments and other departments existed. First, it can select a department randomly, and select a node in it according to probability (1) as the side of edge, and the other side of the edge located in the other department selected randomly, and its probability is still (1). Repeat this process $m_{4}$ times. 
Model Construction. Through analyzing the evolution of organization, we use the method of mean-field to construct model.

(1) To create a new department with probability $p$. In this case, the tissue distribution of internal node ${ }^{i}$ pre-existed do not change with time, Because the new generation of employees within the organization did not interact with staff in other departments .Therefore,

$$
\left(\frac{\partial k_{i}}{\partial t}\right)=\mathbf{O}
$$

(2)To add a new staff to a particular department $\Omega$.

$$
\left(\frac{\partial k_{i}}{\partial t}\right)=\frac{m_{1}}{m+t P} \sum_{j \in \Omega}^{k_{i}} k_{j}
$$

(3)With the business increasing, particular sector will add $m_{2}$ edges randomly. then,

$$
\left(\frac{\partial k_{i}}{\partial t}\right)=\frac{m_{2}}{m+t P}\left[\frac{1}{N_{\Omega}(t)}+\left(1-\frac{1}{N_{\Omega}(t)}\right) \frac{k_{i}}{j \in \Omega} k_{j}\right]
$$

(4)With the reduction of certain business, particular sector will reduce $m_{3}$ edges randomly. Then,

$$
\left(\frac{\partial k_{i}}{\partial t}\right)=-\frac{m_{3}}{m+t p}\left[\frac{1}{N_{\Omega}(t)}+\left(1-\frac{1}{N_{\Omega}(t)}\right) \frac{k_{i}}{\sum_{j=\Omega} k_{j}}\right]
$$

(5) In the existing departments, to add $m_{4}$ edges between the every two departments

$$
\left(\frac{\partial k_{i}}{\partial t}\right)=\left[\frac{2 m_{4}}{m+t p} \sum_{j \in k_{i}}^{k_{j}}-\frac{m_{4}}{(m+t P)^{2}} \sum_{j=0}^{k_{i}} k_{j}\right]
$$

$N_{\Omega}(t)$ is the number of staff in department $\Omega$. The $t$ step, the sum of any sector connectivity in network average is: $\sum_{j \in \Omega} k_{j}=2 t\left(p e_{0}+m_{1}+m_{2}-m_{3}+m_{4}\right) /(m+t p)$

The nodes average in every department is: $N_{\Omega}(t)=m_{0}+t /(m+t p)$

When $t$ is very large and $m_{\text {o }}$ is very small, in order to calculate facilitate, we make $N_{\Omega}(t)=N_{\Omega}(t)-1=t /(m+t p)$ and $c=2\left(p e_{0}+m_{1}+m_{2}-m_{3}+m_{4}\right)$, for the larger $t$, we can get it by $(2) \sim(8)$ :

$\frac{\partial k_{i}}{\partial t}=\frac{m_{1}}{c} \frac{k_{i}}{t}+\frac{m_{2}}{t}+\frac{m_{2} k_{i}}{c t}-\frac{(m+t p) k_{i} m_{2}}{c t^{2}}-\frac{m_{3}}{t}-\frac{m_{3} k_{i}}{c t}+\frac{(m+t p) k_{i} m_{3}}{c t^{2}}+\frac{2 m_{4}}{c} \frac{k_{i}}{t}-\frac{m_{4}}{(m+t p) c} \frac{k_{i}}{t}=\left[\frac{m_{1}}{c}+\frac{m_{2}}{c}-\frac{m_{3}}{c}+\frac{2 m_{4}}{c}-\frac{m_{4}}{(m+t p) c}\right] \frac{k_{i}}{t}+\left(m_{2}-m_{3}\right) \frac{1}{t}$

To make $\frac{m_{1}}{c}+\frac{m_{2}}{c}-\frac{m_{3}}{c}+\frac{2 m_{4}}{c}-\frac{m_{4}}{(m+t p) c}=a$ and $m_{2}-m_{3}=b$, then, $\frac{\partial k_{i}}{\partial t}=a \frac{k_{i}}{t}+b \frac{1}{t}$

From the initial conditions $k_{i}\left(t_{i}\right)=m_{1}$, We can get result as follows: $k_{i}(t)=-\frac{b}{a}+\left(m_{1}+\frac{b}{a}\right)\left(\frac{t}{t_{i}}\right)^{a}$

In unit time, from the above the evolution of the network, we can get the ${ }^{t_{i}}$ probability density is $P_{i}\left(t_{i}\right)=1 /[3 m+t(1+2 p)]$, so 


$$
P\left(k_{i}(t)<k\right)=P\left[t_{i}>\left(\frac{m_{1}+b / a}{k+b / a}\right)^{1 / a} t\right]=1-\frac{1}{[3 m+t(1+t p)]}\left(\frac{m_{1}+b / a}{k+b / a}\right)^{1 / a} t
$$

Then, the degree distribution $P(k)$ is: $P(k)=\frac{t}{a[3 m+t(1+2 p)]}\left(m_{1}+b / a\right)^{1 / a}(k+b / a)^{-\lambda}$

We can obtain the degree distribution of the organization by above analysis, this distribution is power law distribution. Therefore, enterprise organizations have the statistical characteristics of small-world networks, when its scale develop to a certain extent, and it is a complex system with both robustness and fragility.

\section{Model Analysis}

Enterprise organization as a complex system, its robustness and fragility which impact on its survival and development directly. The above used research methods for complex network to construct the model and analyze the complex systems-enterprise organization, and study its robustness by studying the robustness of complex networks. The robustness of complex networks depends on each node overall the network. Though complex network influenced by varieties of internal and external system factors in its evolution, but nothing more than "random attack" and "deliberate attack" two cases. "Random attack" refers to remove nodes from network, "deliberate attack" refers to remove higher network moderate nodes directly[3]..The robustness of enterprise organization refers to the characteristics of their networks after random attacks and deliberate attacks, generally, we can study the network connectivity through the shortest path and the largest Unicom sub-graph of the network[4].

Random attack. Assuming that the enterprise organization suffered from a variety of random attack, $\quad P$ is the proportion of failed nodes number account for all of the network nodes. At this time, the degree of node $k_{0}$ is $k$, because its neigh bore nodes attacked in original network. Well, the original distribution degree after attacked will become:

$$
P(k)=\sum_{k_{0}=k}^{\infty} P_{0}\left(k_{0}\right)\left(\begin{array}{l}
k_{0} \\
k
\end{array}\right)(1-p)^{k} P^{k_{0}-k}
$$

Now, the Average degree $\langle k\rangle$ and $\left\langle k^{2}\right\rangle$ of $P(k)$ are respectively: $\langle k\rangle=\sum_{k_{0}=k}^{\infty} P(k) k=(1-p)\left\langle k_{0}\right\rangle$

$$
\text { and }\left\langle k^{2}\right\rangle=\sum_{k_{0}=k}^{\infty} P(k) k^{2}=(1-p)^{2}\left\langle k_{0}{ }^{2}\right\rangle+p(1-p)\left\langle k_{0}\right\rangle
$$

And $\left\langle k_{0}{ }^{2}\right\rangle$ can be calculated by the distribution degree of network. By $\kappa=\frac{\left\langle k^{2}\right\rangle}{\langle k\rangle}=2$

We can get (18): $\kappa \equiv \frac{\left\langle k^{2}\right\rangle}{\langle k\rangle}=\frac{(1-p)^{2}\left\langle k_{0}{ }^{2}\right\rangle+p(1-p)\left\langle k_{0}\right\rangle}{(1-p)\left\langle k_{0}\right\rangle}=2$

$$
\text { After finishing we can get }(19):^{1-p_{c}=\frac{1}{\kappa_{0}-1}}
$$

Among the indexes, $\kappa_{\mathrm{o}} \equiv\left\langle\boldsymbol{k}_{\mathrm{o}}{ }^{2}\right\rangle /\left\langle\boldsymbol{k}_{\mathrm{o}}\right\rangle$ can get by calculating in original network before attacked by the random, $p_{c}$ is the value of the critical point, we can get (20)from the nature of power law distribution: 
$\kappa \equiv\left(\frac{2-\lambda}{3-\lambda}\right) \frac{K^{3-\lambda}-m^{3-\lambda}}{K^{2-\lambda}-m^{2-\lambda}}$

$m$ and $K$ are the minimum and maximum values of the power law distribution node ,when $m<<K$,we can get $\quad(21)$

$$
\begin{aligned}
& \text { If } \lambda>3 \text {, then } 1-p_{c} \approx\left(\frac{\lambda-2}{\lambda-3} m-1\right)^{-1} ; \\
& \text { If } 2<\lambda<3 \text {, then } 1-p_{c} \approx\left(\left|\frac{2-\lambda}{3-\lambda}\right| m^{\lambda-2} K^{3-\lambda}\right)^{-1} ; \\
& \text { If } 1<\lambda<2 \text {, then } 1-p_{c} \approx\left(\frac{\lambda-2}{\lambda-3} K-1\right)^{-1}
\end{aligned}
$$

When $\lambda>3$, to $p>p_{c}$, network will be completely destroyed. When $\lambda<3$, the $P_{c}$ threshold is larger, so network has strong robustness.

Deliberate attack. Network usually occur the following two conditions under deliberate attacks : (1)the node maximum $K$ in network will turn into $K^{\prime}$ after deliberate attacks, $K^{\prime}<K$;(2) the number of nodes in network maintain unchanged,but the node with high degree subject attacks, the number of edges which connect these nodes will reduce greatly, at this point the network will no longer obey a power law distribution.

First, we take

the phenomenon of talented employees resign due to internal reasons. Before the network is under attack,

$$
\sum_{k=K}^{\infty} P(k)=\frac{1}{N}
$$

After the network is under attack,

$$
\sum_{k=K^{\prime}}^{K} P(k)=\sum_{k=K^{\prime}}^{\infty} P(k)-\frac{1}{N}=P
$$

After finishing and deformation ,we can obtain the available values of $K^{\prime}$ is:

$$
K^{\prime}=m p^{1 / 1-\lambda}
$$

We take into account another condition, that is business leaders can not do their job after they occupy more staff with more resource in organization, then result in a large number of resource flows fracture. To set $p^{\prime}$ is probability of the node with higher degree which delete edges, then it is equal to the ratio of the edge cut and the sum of all the edges, that is:

$$
p^{\prime}=\sum_{k=K^{\prime}}^{K} \frac{k P(k)}{\left\langle k_{\mathrm{o}}\right\rangle}
$$

(27) $\left\langle k_{0}\right\rangle$ is the average degree of the original network. If neglect $K$, for $\lambda>2$, we will obtain:

$$
p^{\prime}=\left(\frac{K^{\prime}}{m}\right)^{2-\lambda}=p^{(2-\lambda) /(1-\lambda)}
$$

Thus, the threshold $p_{c}$ is sensitive to $m$, and the value $m$ becomes larger, its robustness of network is higher. Therefore, it is mainly the junior employees of enterprise organization which can 
decide the robustness size of it. For the enterprise organizations with good spirit of cooperation, If you can give full play to the creative spirit of each of their employees, respecting each employee's opinion, then this organization is more innovative capacity and greater stability.

\section{Conclusion}

The robustness of enterprise organization is essential to the enterprise's survival and development. This paper used the method of complex networks and try to analyze the evolution of enterprise organization by constructing models from a new perspective and made derivation and research the robustness and fragility of enterprise organization. From above study, the conclusion can be drawn as follows:

(1)Enterprise organization is a scale-free network, and obey a power law distribution, when it develop to a certain scale, Thus it has the statistical characteristics of small-world networks[5], that is links between the nodes of the organization have uneven distribution seriously; but this scale-free network has high robustness for random attacks, while also shows strong vulnerability for vandalism. Therefore, In order to improve the organization robustness regarding to vandalism, some measures can be taken effectively, such as changing the network formation mechanism, strengthening network maintenance and eliminating its un-scaled characteristics.

(2) The stability of business organization depends largely on the number of junior staff interaction. If the organization has a good team spirit, gives full play to the initiative and creative spirit of each of its employees, respects the views of each staff, it could maintain high stability regardless of the attack strategy. Therefore, managers should strive to create a good atmosphere of teamwork, giving full play to democracy, only in this way can it make the organization more innovative and stability.

\section{Acknowledgements}

This paper is one of high-level university's key disciplines construction projects of Harbin University of Commerce, China, and that is phased results of innovation management and competitive advantage .This research is supported by humanities and Social Sciences Project of education department in Heilongjiang Province, project No.2013WK0138; and it is supported by research project of doctor of Harbin University of Commerce simultaneously, project No.14rw14

\section{References}

[1] Pollack H N, Li Pingping translation. Uncertain science and uncertain world. Shanghai Science and Education Press,(2005)

[2] Scott D. Novak D. Aultman--Hall. Network robustness index: A new method for identifying critical links and evaluating the performance of transportation networks. Journal of Transport Geography.3.14,(2006)

[3] Curlson. J M, Doyle J. Complexity and robustness. Proceedings of the National Academy of Science. January 13-15,(2002)

[4] Jie Jing. International academic research on the robustness. Journal of Systems Engineering.2.20,(2005)

[5] Qin Xiaohong, Huang Guangqiu. Analysis of marketing network robustness based on the theory of complex network. Business Studies.11,(2010) 